\title{
Routine quantification of rheumatoid factor by rate nephelometry
}

\author{
P J ROBERTS-THOMSON, R McEVOY, T LANGHANS AND J BRADLEY \\ From the Department of Clinical Immunology, Flinders Medical Centre, South Australia 5042
}

SUMMARY In a cross-sectional study of over 3000 consecutive serum specimens the levels of rheumatoid factor (RF) measured by rate nephelometry (Beckman ICS II) were compared with values obtained by the more traditional methods of sheep cell agglutination (Rose-Waaler) and latex agglutination. Similar values for sensitivity and specificity were found for all three methods for rheumatoid arthritis, with nephelometry giving slightly higher levels of sensitivity for other rheumatic disorders. A significant correlation $(\mathrm{r}=0.46, \mathrm{p}<0.01)$ was found between the nephelometric and Rose-Waaler method for 147 consecutive seropositive specimens. Of interest, however, several disparate results were observed, and explanations for these were sought. Longitudinal studies of RF were performed in 49 seropositive patients over a two-year period. The nephelometric method was considered superior compared with the other techniques because of its ability to detect changes in absolute levels at earlier stages and its low interassay coefficient of variance $(11 \%)$.

We conlcude that the nephelometric technique appears suitable for routine diagnostic use, offers several advantages compared with more traditional methods, and is no more expensive per test specimen than the Rose-Waaler technique.

Traditionally, rheumatoid factor (RF) has been measured in the routine diagnostic laboratory by the latex agglutination test and by methods utilising sheep cell agglutination (e.g. Rose-Waaler). These tests are semiquantitative in that they require a subjective interpretation of an end point and have poor reproducibility (e.g. coefficient of variance approximately $20 \%^{1}$ ). In addition the absolute level of RF has to be changed by over $50 \%$ before there is a change in titre if a doubling dilution technique is used. Nevertheless these agglutination tests have become established as the methods most commonly used at the present time to measure $\mathrm{RF}$ in the diagnostic laboratory.

Over the last five years nephelometric methods have been described to measure RF. ${ }^{1-7}$ Nephelometry is a simple and sensitive technique for measuring the degree of light scatter caused by the

Accepted for publication 17 December 1984.

Correspondence to Dr P J Roberts-Thomson, Department of Clinical Immunology, Flinders Medical Centre, Bedford Park, South Australia 5042 interaction of RF with heat aggregated human IgG. There are two principal forms of nephelometric analysis: (1) end point nephelometry, which measures the final light scatter after a fixed period of reaction time, e.g., $60 \mathrm{~min}$, and is dependent on background scatter; ${ }^{4} 5$ and (2) rate nephelometry, which measures the initial rate of scatter and hence is relatively independent of background scatter and results can be obtained within seconds. ${ }^{37}$ There are several advantages in using nephelometric techniques, particularly the latter form, in measuring levels of RF; it is fast, truly quantitative, has a desirably low coefficient of variance, and has been reported to have a low false positive rate in the elderly non-rheumatoid population. ${ }^{13}$ Of the seven published reports concerning the nephelometric RF assay all have concluded that this assay measures RF accurately, and two ${ }^{12}$ have specifically recommended it in preference to the established agglutination techniques.

In the present study we have undertaken a large comparative and analytical study to determine the usefulness of the nephelometric technique in 
measuring $\mathrm{RF}$ in the routine diagnostic laboratory. Cross-sectional and sequential studies of RF levels in over 3000 consecutive specimens submitted to our laboratory were performed, and the results confirmed our initial favourable opinions of the nephelometric method. ${ }^{7}$

\section{Materials and methods}

\section{SERA}

$\mathrm{RF}$ was measured in over 3000 consecutive serum samples received by our laboratory over a two-year period in which a request for RF estimation had been made. The sera were assigned to various diagnostic categories, the diagnosis being assumed to be that clearly identified by the referring source, and in approximately $80 \%$ of instances this referral was from the rheumatology and immunology clinics. In many instances RF was quantified in the same patient on multiple occasions, and 49 patients with rheumatoid arthritis (RA) were identified in whom RF had been measured on five or more occasions. Sera were also obtained from 50 blood donors.

Serum samples were stored at $-20^{\circ} \mathrm{C}$, and $\mathrm{RF}$ estimations were performed twice weekly.

\section{RF ASSAYS}

$\mathrm{RF}$ was measured in all sera by three different assays-namely, the slide agglutination test (Behring Rapi-Tex), sheep cell agglutination (RoseWaaler), and rate nephelometry (Beckman ICS II). The latex agglutination test was performed according to the manufacturer's instructions at a final serum dilution of $1: 10$ and $1: 50$ in diluting buffer, and agglutination was detected visually after gentle mechanical rocking at room temperature for two minutes. The Rose-Waaler sheep cell agglutination test was performed in accordance with published methods, ${ }^{8}$ and a positive titre was defined as $1: 32$ or greater. The rate nephelometric test was performed on a Beckman ICS II with Beckman reagents exactly as described by the manufacturer's technical brochure. The rate and total scatter were also recorded graphically on a Rikadenki dual channel chart recorder connected to the Beckman ICS II instrument via two outputs (labelled 'rate' and 'scatter') on the back panel of the instrument. RF was also measured in some sera and column fractions with a Behring laser nephelometer as previously described. ${ }^{4}$

\section{COLUMN CHROMATOGRAPHY}

Selective sera were fractionated on a $90 \times 2.5 \mathrm{~cm}$ Sepharose 6B (Pharmacia) column, and RF was measured in the eluate by laser nephelometry.
Results

Over a two-year period RF was measured in 3000 ? consecutive specimens received by our laboratory음 For all specimens RF was measured by three methods, and negative and positive RF controls were included in each assay batch. For the nephe $\frac{\mathbb{2}}{2}$ lometric assay the coefficient of variance of the positive control was $11.0 \%$ over the two-year periodand for a $6 / 12$ month period it was $6 \cdot 1 \%$. In 29 . specimens (i.e. $<1 \%$ of total) it was not possible to record a RF level accurately because of excess background scatter due to gross lipaemia.

The nephelometric RF level measured in 50 bloods donors is seen in Fig. 1. Four individuals had levels $>80 \mathrm{IU} / \mathrm{ml}$, and this level was arbitrarily chosen asio the upper limit of normal in our non-hospitake 'healthy' population. The RF levels found in various? disease categories are shown in Fig. 1. As predictedthe greatest prevalence and highest values were ${ }^{\complement}$ found in RA, and $80 \%$ of our rheumatoid patients were seropositive by the nephelometric assay.

The sensitivity and specificity of the three assayso are given in Table 1 . In general the methods gave similar results; the nephelometric assay was slightlyo more sensitive than the agglutination assays but as little less specific (than the Rose-Waaler assay). The cost of each assay (cost of reagents, technicians time etc.) is also shown in this table. A comparison of the RF levels measured by the nephelometric and $\overrightarrow{\vec{b}}$ Rose-Waaler assays for 359 random consecutive ${ }^{3}$ specimens is shown in Fig. 2. Excluding the

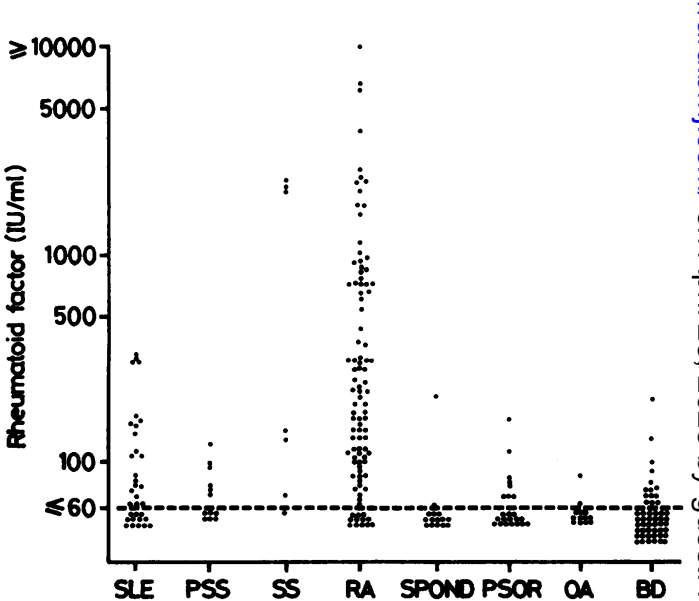

Fig. 1 RF levels $(I U / \mathrm{ml})$ measured by rate nephelometry in patients with rheumatoid arthritis $(R A)$, systemic lupus erythematosus (SLE), progressive systemic sclerosis (PSS), Sjögren's syndrome (SS), spondylarthritis (SPOND), psoriatic arthritis (PSOR), or osteoarthritis $(O A)$ and in 50 blood donors $(B D)$. 
Table 1 Sensitivity and specificity of $R F$ assayed by three methods

\begin{tabular}{|c|c|c|c|c|c|c|c|c|c|c|}
\hline & $B D^{*}$ & $R A$ & $S L E$ & PSS & $S S$ & SPOND & PSOR & $P M R$ & $O A$ & $\begin{array}{l}\text { Cost } \\
\text { (\$) }\end{array}$ \\
\hline No. & 50 & 100 & 33 & 12 & 7 & 14 & 23 & 19 & 14 & - \\
\hline Neph. $>80 \mathrm{IU} / \mathrm{ml}$ & 4 & 80 & 13 & 4 & 5 & 1 & 4 & 1 & 1 & 1.60 \\
\hline Latex (1:10 dil) & 2 & 83 & 8 & 3 & 4 & 1 & 2 & 2 & 0 & $0 \cdot 8$ \\
\hline Rose-Waaler $>1: 32$ & 0 & 75 & 6 & 2 & 4 & 1 & 1 & 1 & 0 & $1 \cdot 60$ \\
\hline
\end{tabular}

${ }^{*} \mathrm{BD}=$ blood donors; $\mathrm{RA}=$ rheumatoid arthritis; $\mathrm{SLE}=$ systemic lupus erythematosus; $\mathrm{PSS}=$ progressive systemic sclerosis; $\mathrm{SS}=\mathrm{Sjögren's}$ syndrome; $\mathrm{SPOND}=$ spondylarthritis; $\mathrm{PSOR}=$ psoriatic arthritis; $\mathrm{PMR}=$ polymyalgia rheumatica; $\mathrm{OA}=0$ osteoarthritis.

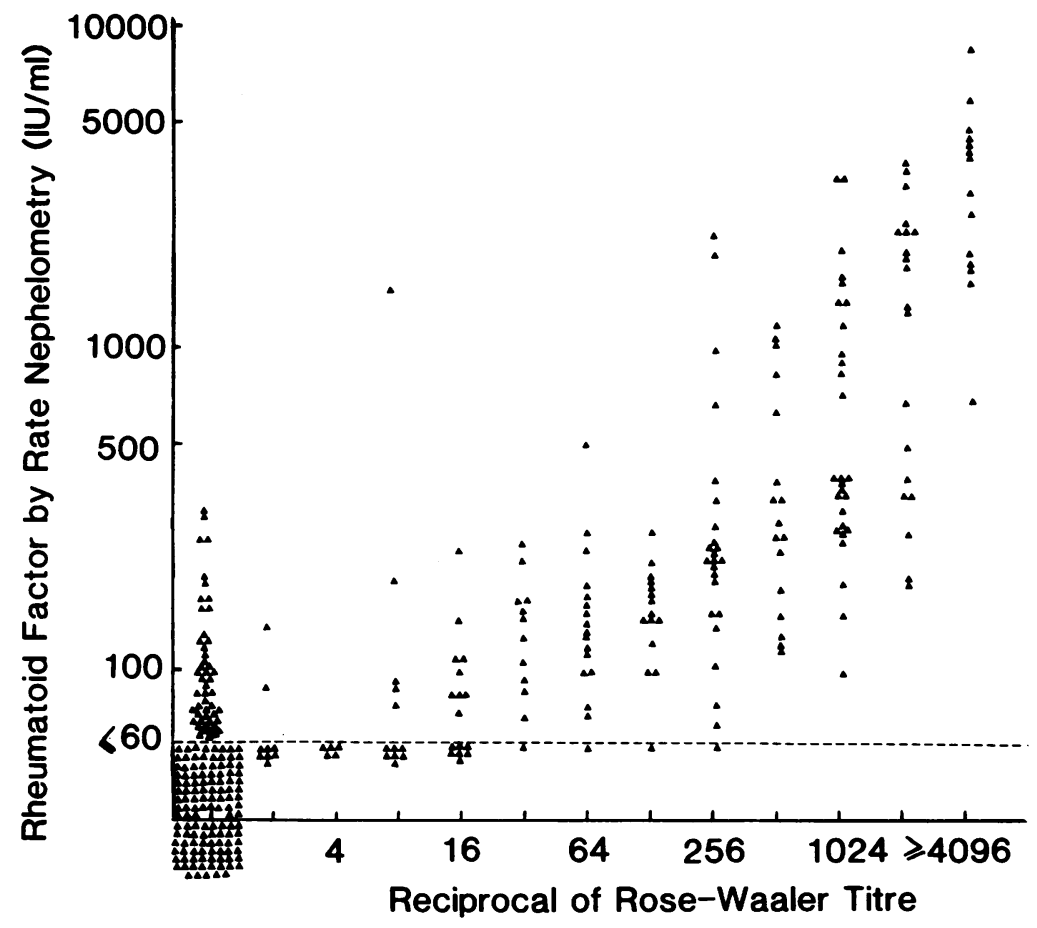

Fig. 2 Comparison of RF levels measured by the Rose-Waaler agglutination assay and expressed as reciprocal of titre ( $x$ abscissa) with RF levels measured by rate nephelometry (y ordinate).

seronegative specimens a significant correlation $(r=0.46, p<0.01)$ was found between the two assays. More importantly, however, a number of disparate results were obtained, and possible reasons for these were examined. In a review of the comparative results for all sera studied over two years six patients were observed who gave a persistently high or very high nephelometric reading and negative or low positive Rose-Waaler titre (Table 2). The diagnoses in these patients were Sjögren's syndrome in three, hypergammaglobulinaemic purpura in two, and mixed cryoglobulinaemia in the other. Sera from three of these
Table 2 High RF by nephelometry with low Rose-Waaler titre

\begin{tabular}{llll}
\hline Patient & Diagnosis & Rose-Waaler titre & $\begin{array}{l}\text { Nephelometric } \\
R F(I U / m l)\end{array}$ \\
\hline 1 & HP* & $1: 16$ & 5340 \\
2 & HP & $1: 128$ & 3560 \\
3 & SS & $1: 256$ & 2140 \\
4 & SS & $1: 128$ & 2090 \\
5 & SS & $1: 256$ & 2240 \\
6 & MC & $1: 8$ & 1590 \\
\hline
\end{tabular}

*HP=hypergammaglobulinaemic purpura; $\mathrm{SS}=$ Sjögren's syndrome; MC=mixed cryglobulinaemia. 


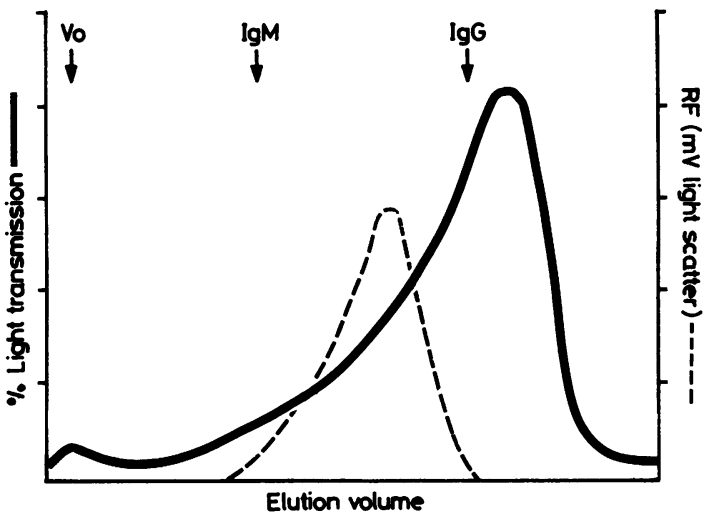

Fig. 3 Sepharose $6 B$ chromatographic profile of serum of a patient with hypergammaglobulinaemic purpura. The eluting positives of the void volume, Vo, IgM, and $\operatorname{IgG}$ are indicated. Note the $R F$ profile elutes maximally between the two immunoglobulin markers.

patients were fractionated by Sepharose 6B chromatography and the eluate examined for RF (Fig. 3). RF peaked in the dimeric IgG position, suggesting that the RF was either $\operatorname{IgG}, \operatorname{IgA}$, or both.

Several sera which gave high Rose-Waaler titres but persistently negative nephelometric values were observed to give rapid and high initial increases in rates of scatter but then dropped back to negative scatter rates, and a negative RF level was finally computed by the instrument. Further dilution of these specimens would then result in high nephelometric RF levels, suggesting that the first value on the initial dilution was incorrect.

RF levels were measured sequentially in 49 patients. The nephelometric RF levels tended to fluctuate less than the Rose-Waaler titres, and gradual declines were seen in response to effective drug treatment (Figs. 4a and b).

\section{Discussion}

Quantification of RF by rate nephelometry appears to be well suited for the routine diagnostic laboratory and has several advantages compared with the more traditional agglutination tests. The nephelometric test is simple, gives rapid results, has good precision, and has the ability to measure $\mathrm{RF}$ in absolute terms. The sensitivity and specificity of this assay are very similar to the agglutination techniques and the method has a similar cost to that of the Rose-Waaler assay. There are some disadvantages: the cost of the nephelometer (although the instrument can be used to measure many other serum proteins, e.g., C-reactive protein), the
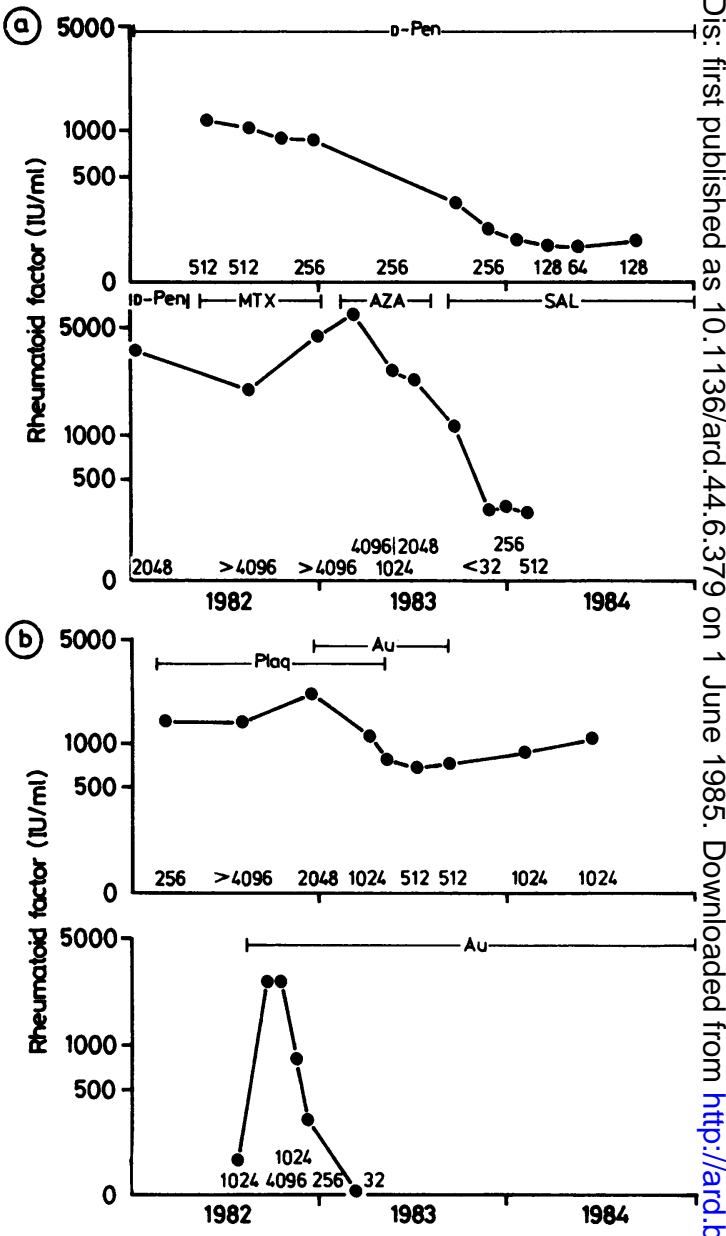

Fig. 4 Sequential studies of $R F(I U / \mathrm{ml})$ measured by rate nephelometry in four patients with $R A$. The reciprocal of the Rose-Waaler titre is indicated by the figures on the $x$ abscissa measured at the same time.

$D$-Pen $=D$-penicillamine $; M T X=$ methotrexate; $A Z A=$ azothioprine; $S A L=$ salazopyrin; $P l a q=$ plaquenil; $A u=$ gold .

inability to measure RF in grossly lipaemic speci 0 mens due to the high levels of background scatter $\tilde{\omega}_{\omega}^{N}$ and the occasional disparate result compared with the agglutination assays. With regard to specimenso giving high nephelometric values and low Rose-市 Waaler titres we have shown that in some instances ${ }^{\infty}$ this is due to the presence of IgG or IgA RF which give poor agglutination. ${ }^{4}$ This discriminatory ability of the nephelometric test can be therefore con $\cong$ sidered as a bonus and can be of diagnostic value (e.g., occurs in some patients with Sjögren'so syndrome, hypergammaglobulinaemic purpura, or 
mixed cryoglobulinaemia). Occasional specimens were observed in which there was a high RoseWaaler titre but a negative nephelometric value. Further measurement at a higher serum dilution would then reveal a high nephelometric value, suggesting that the initial nephelometric value was incorrect. In studying these specimens we noted that an early rapid increase in scatter was initially detected followed by a rapid decline to zero or negative scatter values, resulting in a normal value being finally computed. Macroscopically it was also apparent that the reaction cell contained large aggregates of immune complexes which dropped rapidly to the bottom of the cell. We now routinely observe the scatter profile with the aid of twin channel chart recorder to detect such specimens. If high initial rates of scatter are recorded with negative final RF values, we automatically measure the RF level again with the next dilution step of serum sample to obtain the correct value. These aberrant scatter recordings are seen in approximately $2 \%$ of specimens. The cause of these initial false negatives is unclear but may relate to (a) bound RF being made available by subsequent dilution of the serum or (b) rapid formation of large aggregates which fall towards the bottom of the reaction cell out of the optical light path.

The nephelometric test appears suitable for following serum RF levels on a sequential basis. Smooth curves were obtained after plotting values on semilog graph paper over time, and early declines of RF were useful in monitoring a patient's response to therapy. In contrast the Rose-Waaler titres tended to fluctuate more widely. As a consequence of our experience we have now discontinued measuring RF by the Rose-Waaler technique but are continuing to screen for RF using latex agglutination at 1:10 serum dilution and measuring its absolute quantity with the rate nephelometric technique.

We are grateful to Mrs M Molnar who prepared the manuscript and has provided expert secretarial assistance during this study.

\section{References}

1 Knight R K, Pritchard M H. Nephelometry compared with differential antibody titre in routine rheumatoid factor measurements. Ann Rheum Dis 1982; 41: 426-9.

2 Pritchard M H, Jobbins K. Nephelometry $v$. differential agglutination titre in the measurement of rheumatoid factors. $J$ Clin Pathol 1981; 34: 396-9.

3 Finley P R, Hicks M J, Williams R J, Hinlicky J, Lichti D A. Rate nephelometric measurement of rheumatoid factor in serum. Clin Chem 1979; 25: 1909-14.

4 Roberts-Thomson P J, Wernick R M, Ziff M. Quantitation of rheumatoid factor by laser nephelometry. Rheumatol Int 1982; 2: 17-20.

5 Virella G, Waller M, Fudenberg H H. Nephelometric method for determination of rheumatoid factor. J Immunol Methods 1978; 22: 247-51.

6 Weinblatt M E, Schur P H. Rheumatoid factor detection by nephelometry. Arthritis Rheum 1980; 23: 777-9.

7 Roberts-Thomson P J, Gale R, McEvoy R, Bertouch J, Gordon $\mathrm{T}$, Bradley J. Rate nephelometric determination of rheumatoid factor (RF) (abstract). Aust NZ J Med 1983; 13: 213.

8 Waller M. Methods of measurement of rheumatoid factor. Ann NY Acad Sci 1969; 168: 5. 